\title{
Improvement of histological biopsy diagnosis of hepatocellular carcinoma by genomic biomarkers?
}

\author{
Rolf Lamerz
}

Screening programmes have been developed for the surveillance of patients at risk of hepatocellular carcinoma (HCC; mainly liver cirrhosis) for the detection at an early stage, ie, suspicious liver nodules not larger than $2 \mathrm{~cm}$ in diameter often detected by ultrasound. Treatment indication needs a clear diagnosis for which one option is a biopsy often hampered by a relevant rate of false negatives and by a risk of bleeding and malignant seeding. Therefore, for the diagnosis of HCC imaging criteria are preferred requiring contrast enhancement and dynamic imaging. The recent update of the American Association for the Study of Liver Diseases (AASLD) guidelines in $2010^{1}$ recommends for a nodule greater than $1 \mathrm{~cm}$ one single imaging technique to be used among CT and MRI, and in the case of non-diagnostic criteria the choice between a second technique or a biopsy.

Establishing HCC diagnosis at an ideal size of less than $2 \mathrm{~cm}$ relates to the transition from low to high grade dysplasia and very early HCC as described by the international working party classification ${ }^{2}$ and a final international diagnostic consensus. ${ }^{3}$ From two types of HCC less than $2 \mathrm{~cm}$, early HCC has a vaguely nodular appearance, is well differentiated and corresponds to the very early HCC stage of the Barcelona Clinic Liver Cancer classification, with the absence of microvascular invasion excluding a clear imaging profile for diagnosis. The progressed HCC has a distinctly nodular pattern, is more moderately differentiated and often with evidence of microvascular invasion. As the diagnostic sensitivity of biopsy may also be limited in very early HCC because of its composition by well differentiated hepatocytes, the additional

Correspondence to Professor Dr Rolf Lamerz, Med. Klinik II, Klinikum Campus Grosshadern,

Ludwigs-Maximilians-Universität, Marchionini-Strasse 15. München 81377, Germany;

rolf.lamerz@med.uni-muenchen.de use of genomic biomarkers has been introduced. According to a study for the diagnosis of early HCC, a 3-gene set obtained a discriminatory accuracy of $94 \%$ with glypican-3 (GPC3) of most predictive power. ${ }^{4}$ GPC $3^{5}$ is a cell-surface heparan sulphate proteoglycan secreted into plasma, its immunoreactivity is cytoplasmic, sometimes membranous or canalicular, seldom only focal. ${ }^{3}$ Another study $^{6}$ identified heat-shock protein 70 (HSP70) as the most abundantly upregulated gene in early HCC components. HSP70 is implicated in the regulation of cell cycle progression, in apoptosis and in tumorigenesis. Its nucleocytoplasmic and mostly focal immunoreactivity is found in most HCC including early and welldifferentiated forms. ${ }^{3}$ Another profiling HCC study ${ }^{7}$ identified one clone as the glutamine synthetase (GS) transcript expressed and tightly regulated during development in the hepatic lobule. In human HCC, an upregulation of GS messenger RNA, protein and activity was described as well as a stepwise increase in GS immunoreactivity from precancerous lesions to early HCC with strong and diffuse staining intensity. ${ }^{5}$

Di Tommaso et al ${ }^{8}$ performed a retrospective immunohistochemistry marker study of HSP70, GPC3 and GS aiming at the distinction of high-grade dysplasia from well-differentiated HCC in 18 to 19-gauge needle liver biopsy specimens comprising large regenerative nodules (13), low-grade (21) and high-grade dysplastic nodules (50), very well-differentiated (17), well-differentiated (40) and moderately poorly differentiated (35) HCC. Using at least two markers positive for the differentiation of HCC from non-malignant nodules, the sensitivity, specificity, positive predictive value and accuracy rates were $58.7 \%, 100 \%, 100 \%$ and $78.4 \%$, respectively, and for differentiating highgrade dysplastic nodules from very welldifferentiated HCC, were still 49.1\%, $100 \%, 100 \%$ and $72.9 \%$, respectively.
In their paper published in Gut, Cai et $a l^{9}$ (see page 967) try to improve the immunohistological diagnosis of HCC in liver biopsies by implementing in addition to the biomarkers HSP70 and GPC3 the molecular marker enhancer of zeste homolog 2 (EZH2) known for its important role in hepatocellular carcinoma tumorigenesis. EZH2 is the histone $\mathrm{H} 3$ lysine 27 methyltransferase of polycombrepressive complex 2 (PRC2), mediates transcription silencing, and has been shown to maintain cell identity, cell cycle regulation and oncogenesis. ${ }^{10}$

The authors investigated retrospectively by immunohistochemistry the expression dynamics of EZH2, HSP70 and GPC3 in two independent surgical testing (212 HCC) and validation (126 HCC, 37 dysplastic nodules) cohorts of HCC patients in order to evaluate their diagnostic yield for HCC detection. The criteria obtained there were then applied to the diagnostic performance of 18-gauge needle biopsies from 72 HCC and 57 different precancerous lesions from nodules less than $3 \mathrm{~cm}$ in diameter of an additional biopsy cohort with consecutively confirmed diagnosis from surgical specimens following operation. By immunohistochemistry analysis the sensitivity and specificity of EZH2 for HCC detection was $95.8 \%$ and $97.8 \%$ in the testing corhort, with lower results in the validation cohort $(83.3 \%, 90.0 \%)$. For the diagnosis of well-differentiated HCC, these rates amounted to $68.9 \%$ and 91.5\% (EZH2), $62.5 \%$ and $98.5 \%$ (HSP70), $50.0 \%$ and $92.1 \%$ (GPC3), and to $75.0 \%$ and $100.0 \%$ for the three marker panel, respectively.

In fine-needle biopsies, positive cases for at least one panel marker consecutively increased from large regenerative nodules $(0 / 6)$ and hepatocellular adenomas (0/6) to focal nodular hyperplasias $(2 / 20,10 \%)$, dysplastic nodules (7/25, 28\%), welldifferentiated HCC (16/18, 88.9\%) and moderately/poorly differentiated HCC (54/54, 100\%). Considering at least two positive panel markers, positive cases were detected in none of the large regenerative nodules/hepatocellular adenomas, focal nodular hyperplasias and dysplastic nodules, but in 11/18 (61.1\%) well-differentiated HCC, 32/37 (86.5\%) moderately differentiated HCC and 15/17 (88.2\%) poorly differentiated HCC. Accordingly, the relevant statistical parameters of sensitivity, specificity, positive predictive value and negative predictive value at a prevalence of $55.8 \%$, accuracy and Youden index $(\mathrm{J})$ values changed from 
$97.2 \%, 84.2 \%, 88.6 \%, 96.0 \%, 91.5 \%$ and 0.81 , respectively, for at least one marker positive to $80.6 \%, 100 \%, 100 \%, 80.3 \%$, $89.2 \%$ and 0.81 , respectively, for at least two positive markers. These findings qualify EZH2 protein as a promising diagnostic biomarker of HCC and the use of the three marker panel as a significant improvement of HCC detection in liver biopsy tissues.

Despite the intriguing study findings some limitations have to be considered: first, the study is of retrospective nature and is thus less reliable than a prospective study. Second, only patients with lesions confidently classified as HCC by biopsy and explant analysis were included in the study, while excluding cases without later operation.

Third, the evaluation criteria of biopsy specimens were derived from the surgically resected specimens of the testing and validation cohorts including a high proportion of large HCC lesions $(60 \%>5 \mathrm{~cm}$ in diameter, $56 \%$ stages III and IV cases), which only afford a haematoxilin-eosin staining for confident histological HCC diagnosis. However, the authors have not specified the size of their surgically resected tumours (or non-tumours) for confirmation of biopsy diagnosis.

It is understood that well-established criteria for an unequivocal HCC diagnosis are also needed for the inclusion of genomic biomarkers and their final validation. Therefore, further prospective studies with a predominant inclusion of HCC lesions of less than $2 \mathrm{~cm}$ in diameter are needed for the final validation and confirmation of the conclusions of this interesting study.

Competing interests None declared.

Provenance and peer review Commissioned; internally peer reviewed.

Published Online First 24 March 2011

Gut 2011;60:881-882. doi:10.1136/gut.2011.238980

\section{REFERENCES}

1. Bruix J, Sherman M. Management of hepatocellular carcinoma: an update. July 2010. http://www.aasld. org/practiceguidelines/Documents/Bookmarked\% 20Practice\%20Guidelines/HCCUpdate2010.pdf (accessed Jul 2010)

2. International Working Party. Terminology of nodular lesions of the liver. Hepatology 1995;22:983-93.
3. International Consensus Group for Hepatocellular Neoplasia. Pathologic diagnosis of early hepatocellular carcinoma: a report of the international consensus group for hepatocellular neoplasia. Hepatology 2009:49:658-64.

4. Llovet JM, Chen $\mathrm{Y}$, Wurmbach $\mathrm{E}$, et al. A molecular signature to discriminate dysplastic nodules from early hepatocellular carcinoma in HCV cirrhosis. Gastroenterology 2006;131:1758-67.

5. Ho M, Kim H. Glypican-3: a new target for cancer immunotherapy. Eur J Cancer 2011:47:333-8.

6. Chuma M, Sakamoto M, Yamazaki K, et al. Expression profiling in multistage hepatocarcinogenesis: identification of HSP70 as a molecular marker of early hepatocellular carcinoma. Hepatology 2003;37:198-207.

7. Christa L, Simon MT, Flinois JP, et al. Overexpression of glutamine synthetase in human primary liver cancer. Gastroenterology 1994;106:1312-20.

8. Di Tommaso L, Destro A, Seok JY, et al. The application of markers (HSP70, GPC3 and GS) in liver biopsies is useful for detection of hepatocellular carcinoma. J Hepatol 2009;50:746-54.

9. Cai MY, Tong ZT, Zheng F, et al. EZH2 protein a promising immunomarker for the detection of hepatocellular carcinomas in liver needle biopsies. Gut 2011;60:967-76.

10. Tsang DPF, Cheng ASL. Epigenetic regulation of signalling pathways in cancer: role of the histone methyltransferase EZHR. J Gastroenterol Hepatol 2011;26:19-27.

\section{Prebiotic carbohydrates: not sweet yet for Crohn's disease?}

\section{Philippe Marteau ${ }^{1,2,3}$}

The double-blind placebo controlled study performed by Benjamin et al ${ }^{1}$ published in Gut (see page 923) shows that the daily consumption of $15 \mathrm{~g}$ fructo-oligosaccharides (FOS) worsens the clinical signs of active Crohn's disease. The message for the clinician and the patient is clear: despite a strong rationale and hopes from open studies, ${ }^{2}$ there is presently no evidence of efficacy of prebiotics in Crohn's disease. The same conclusion applies for probiotics and synbiotics. ${ }^{3}$ However, one should avoid throwing the baby out with the bath water.

\footnotetext{
${ }^{1}$ Université Denis Diderot, Paris 7, France; ${ }^{2}$ Laboratoire de Biologie EA 3199, CNAM, Paris, France; ${ }^{3}$ AP-HP, Hôpital Lariboisière, Paris, France

Correspondance to Dr Philippe Marteau, Département Médico-chirurgical de Pathologie Digestive, Hôpital Lariboisière, 2 rue Ambroise Paré, 75475 Paris cedex, France; philippe.marteau@lrb.aphp.fr
}

Descriptive studies of the microbiota and microenvironment in Crohn's disease have repeatedly shown dysbiosis, which is probably partly a consequence of the ecological modifications induced by the disease but also a worsening factor. ${ }^{4}$ It is thus important to obtain knowledge on both the positive and detrimental consequences of manipulating the microbiota in the various clinical situations of the disease. ${ }^{3}$

FOS are non-digestible oligosaccharides, and as such exhibit an osmotic effect and increase fermentation (gas and short chain fatty acids and low $\mathrm{pH}$ at least in the right colon). ${ }^{6}$ Moreover, they usually have prebiotic properties, especially a bifidogenic effect (ie, they increase concentrations of bifidobacteria in the colonic lumen). ${ }^{6}$ These metabolic characteristics (osmotic effect and fermentation) explain their potential risks of increasing gas production, bloating, abdominal pain and diarrhoea. ${ }^{5}$ The osmotic effect depends on the chain length and the results obtained here with synergy1 may not apply to other FOS with a longer chain length. The bet for any trial is the hope that, at the chosen dose, efficacy would overweight those predictable dose-dependent side effects.

Clinicians must keep on advising patients with inflammatory bowel disease complaining of bloating or diarrhoea to restrict their consumption of nondigestible oligosaccharides (lactose in milk, sugar alcohols and FOS). ${ }^{7}$ They should explain diet and correct any confusing information (often coming from the internet) including advertisements for products without any clinical study. The probiotic Lactobacillus rhamnosus GG is an example in which optimistic open studies were followed by well-designed negative randomised controlled trials in patients. ${ }^{89}$

The study by Benjamin et a ${ }^{1}$ also brings important new facts to the researcher and raises new questions. Indeed, FOS did not exhibit a bifidogenic effect and this contrasts with trials in healthy subjects in whom FOS at this dose is usually well tolerated and has a bifidogenic effect. ${ }^{6}$ Why was that? Is it just because the endogenous levels of bifidobacteria were already quite high in the patients? ${ }^{1} 6$ A short colonic transit time may also play a role. 


\title{
GUT Improvement of histological biopsy diagnosis of hepatocellular carcinoma by genomic biomarkers?
}

Rolf Lamerz

Gut 2011 60: 881-882 originally published online March 24, 2011

doi: $10.1136 /$ gut.2011.238980

Updated information and services can be found at:

http://gut.bmj.com/content/60/7/881

\begin{abstract}
These include:
References This article cites 9 articles, 1 of which you can access for free at: http://gut.bmj.com/content/60/7/881\#BIBL

Email alerting

Receive free email alerts when new articles cite this article. Sign up in the service box at the top right corner of the online article.
\end{abstract}

\section{Notes}

To request permissions go to:

http://group.bmj.com/group/rights-licensing/permissions

To order reprints go to:

http://journals.bmj.com/cgi/reprintform

To subscribe to BMJ go to:

http://group.bmj.com/subscribe/ 